\title{
Surgery untying of coloured knots
}

\author{
DANIEL MOSKOVICH
}

For $p=3$ and for $p=5$ we prove that there are exactly $p$ equivalence classes of $p$-coloured knots modulo \pm 1 -framed surgeries along unknots in the kernel of a $p$-colouring. These equivalence classes are represented by connect-sums of $n$ left-hand $(p, 2)$-torus knots with a given colouring when $n=1,2, \ldots, p$. This gives a 3-colour and a 5-colour analogue of the surgery presentation of a knot.

57M25; 57M10, 57M27

\section{Introduction}

A $p$-colouring of a knot $K$ is a surjective homomorphism $\rho$ from its knot group $\mathfrak{G}:=\pi_{1}\left(S^{3}-K\right)$ to $D_{2 p}:=\left\{t, s \mid t^{2}=s^{p}=1, t s t=s^{p-1}\right\}$ the dihedral group of order $2 p$, when $p$ is any odd integer. The pair $(K, \rho)$ is called a $p$-coloured knot. It is a well-known fact that we can encode $\rho$ as a colouring of arcs of a knot diagram by elements of $\mathbb{Z}_{p}$ (the cyclic group of order $p$ ), subject to the 'colouring rule' that at least two colours are used, and that at each crossing half the sum of the labels of the under-crossing arcs equals the label of the over-crossing arc modulo $p$. By labeling an arc by an element $n \in \mathbb{Z}_{p}$, we are indicating that $\rho$ maps its meridian to the element $t s^{n} \in D_{2 p}$. If a knot $K$ admits such a colouring the knot is said to be $p$-colourable, and $\rho$ is said to be a $p$-colouring of $K$. These definitions may be extended to links and to tangles. A necessary and sufficient condition for a knot to be $p$-colourable is that its determinant be divisible by $p$. Whether or not a knot is $p$-colourable is the simplest non-trivial invariant which detects non-commutativity of the knot group. For more about $p$-colourability we refer the reader to Fox's original paper [4].

In this paper we investigate the concept of untying a $p$-coloured knot by \pm 1 -framed surgery. Any knot may be untied by surgery along \pm 1 -framed null-homotopic unknots. But such surgeries may not preserve $p$-colourability. The remedy to this is only to allow surgeries that preserve a $p$-colouring $\rho$ of $K$ - surgeries by \pm 1 -framed loops in the kernel of $\rho$. We call such operations surgery in $\operatorname{ker} \rho$.

It is natural to ask what the equivalence classes are of $p$-coloured knots modulo such surgeries. Our main theorem is: 
Theorem 1 There are exactly $p$ equivalence classes of $p$-coloured knots modulo surgery in $\operatorname{ker} \rho$ for $p=3,5$. These equivalence classes are represented by connectsums of $n$ left-hand $(p, 2)$-torus knots with a given colouring, for $n=1,2, \ldots p$.

The above theorem tells us in particular that any $p$-coloured knot $(K, \rho)$ is equivalent modulo $\operatorname{ker} \rho$ to a connect-sum of $n$ left-hand $(p, 2)$-torus knots with a given colouring, with some $n=1,2, \ldots p$.

That there are at most $p$ equivalence classes of $p$-coloured knots modulo surgery in $\operatorname{ker} \rho$ is proved by taking a disc-band presentation of the knot and first unlinking the bands (Section 3) to get a connect-sum of genus 1 knots. The number of twists in each of the bands may then be $p$-reduced, and the remaining knots may be reduced to $p$-coloured $(p, 2)$-torus knots explicitly. The final stage is to reduce the number of connect-summands. These steps are carried out in Section 4 for $p=3$ and in Section 5 for $p=5$. That there are at least $p$ such equivalence classes is proved in Section 6 by finding a non-trivial invariant of $p$-coloured knots which is invariant under surgery in ker $\rho$. Connect-sums of $n$ copies of a $(p, 2)$-torus knot with a given colouring are separated by this invariant, for $n=1,2, \ldots p$.

Conjecture 1 The invariant of Section 6 is an complete invariant on the set of equivalence classes modulo ker $\rho$ for any odd prime $p$. In other words Theorem 1 holds any odd prime $p$.

It is interesting to consider what the corresponding conjecture should be when $p$ is not prime.

Note that the converse of this conjecture is easy- if $K$ is a $p$-coloured knot with $p$-colouring $\rho$ and $K^{\prime}$ is a knot obtained from $K$ by surgery by an element $C$ in $\operatorname{ker} \rho$, then $K^{\prime}$ is $p$-colourable with $p$-colouring $\rho^{\prime}$ induced by $\rho$. A presentation for the fundamental group of $S^{3}-K^{\prime}$ is given by the Wirtinger presentation of $K \cup C$ (see Kawauchi [7]), where the relations added to the set of generators of $\pi_{1}\left(S^{3}-K\right)$ when we push it forward by the surgery map into $\pi_{1}\left(S^{3}-K^{\prime}\right)$ are in the kernel of $\rho$. Thus $K^{\prime}$ is $p$-colourable, with the representation $\rho^{\prime}$ induced by the image of $\rho$ on the push-forward of the generators of $\mathfrak{G}$.

Two applications of Theorem 1 are given in Section 7.

Firstly, as pointed out to the author by Andrew Kricker, a surgery presentation for $3-$ and 5-coloured knots gives a surgery presentation for irregular branched dihedral coverings of knots associated to the dihedral groups $D_{6}$ and $D_{10}$. In this way they form the basis for research into invariants of $p$-coloured knots in the spirit of Garoufalidis and Kricker's work [5; 6]. In [9], the non-commutative surgery presentation of 
coloured knots given in this paper is used to define a non-commutative analogue of the Kontsevich invariant for 3- and 5-coloured knots. Examining the loop expansion of such a Kontsevich invariant is expected to provide dihedral analogues of such classical invariants as the Alexander polynomial.

A second application of Theorem 1, which was pointed out to the author by Makoto Sakuma, is that for a certain class of 3-manifolds they enable us to expand a result of Przytycki and Sokolov [10] (see also Sakuma [11]) concerning surgery presentations of periodic 3-manifolds to the case of dihedral periods. It is an interesting problem to expand these results further to a wider class of manifolds and periods.

\section{Acknowledgements}

This paper owes a huge amount to a number of people. The author would like to sincerely thank Tomotada Ohtsuki for his support and encouragement, for suggesting substantial simplifications to some of the proofs and reorganization of the material, for pointing out various minor gaps, and for his topological interpretation of the invariant in Section 6. The author would also like to thank Andrew Kricker for providing motivation for the research that lead to this paper and for his valuable comments and advice throughout. Special thanks also to Makoto Sakuma for most useful discussions including pointing out a gap in an earlier version of the proof of Proposition 7, to Kazuo Habiro for important comments, encouragement and advice, especially for pointing out an error in Section 5 in a previous version, and to Steven Wallace for his careful reading and useful comments. The author would finally like to thank the anonymous referee for numerous comments and suggestions for improvements.

\section{Basic moves}

We begin by identifying moves which relate $p$-coloured tangles $(T, \rho)$ through surgery in $\operatorname{ker} \rho$.

Notation If $(T, \rho)$ and $\left(T^{\prime}, \rho^{\prime}\right)$ are related by a sequence of \pm 1 -framed surgeries by components in $\operatorname{ker} \rho$, we write $(T, \rho) \sim\left(T^{\prime}, \rho^{\prime}\right)$.

Notation It is convenient to denote half twists by numbers in boxes. By convention, we set:
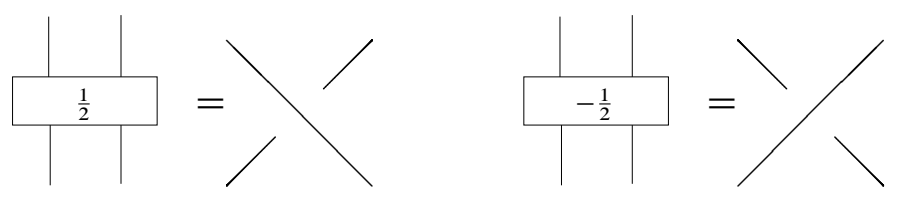

Algebraic 83 Geometric Topology, Volume 6 (2006) 
The next proposition lists some basic moves which we would like to use in our proofs.

Proposition 2 The $R R$ move:

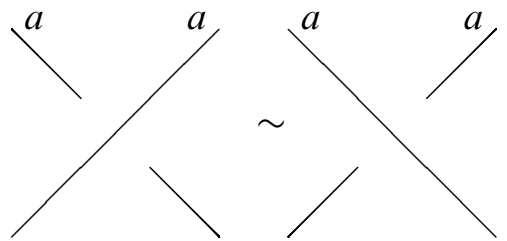

The $R 2 G$ move:

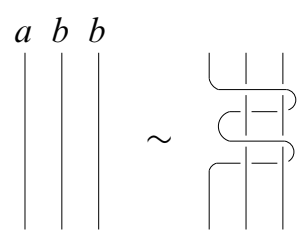

Proof Note that since an element $t s^{i}$ is its own inverse, we may ignore the orientation of the strands. Changing the orientation of the surgery component reverses linkage. The $R R$ move is the result of 1 -framed surgery on a component which loops around both strands.

The $R 2 G$ move is the result of 1 -framed surgery on a component which loops twice around the strand labeled $a$ and once around the two stands labeled $b$. Various $R R$ 's are necessary to clean up. Graphically:
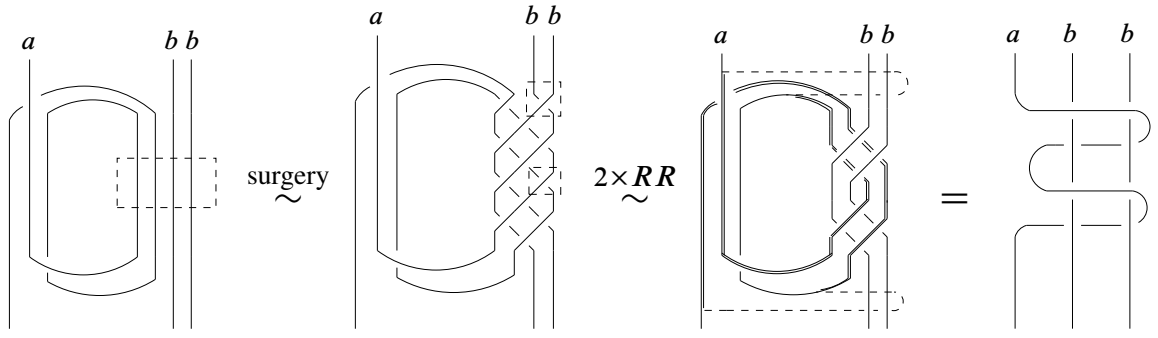

This completes the proof.

The following move, which is a combination of $R R$ and of $R 2 B$, is also useful.

Corollary 3 ( $L T$ move)

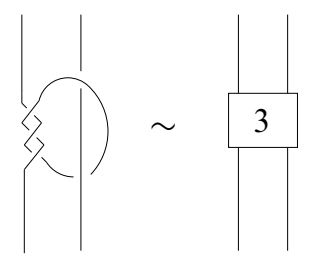

Algebraic 83 Geometric Topology, Volume 6 (2006) 
Proof The colours below are to be read modulo $p$.
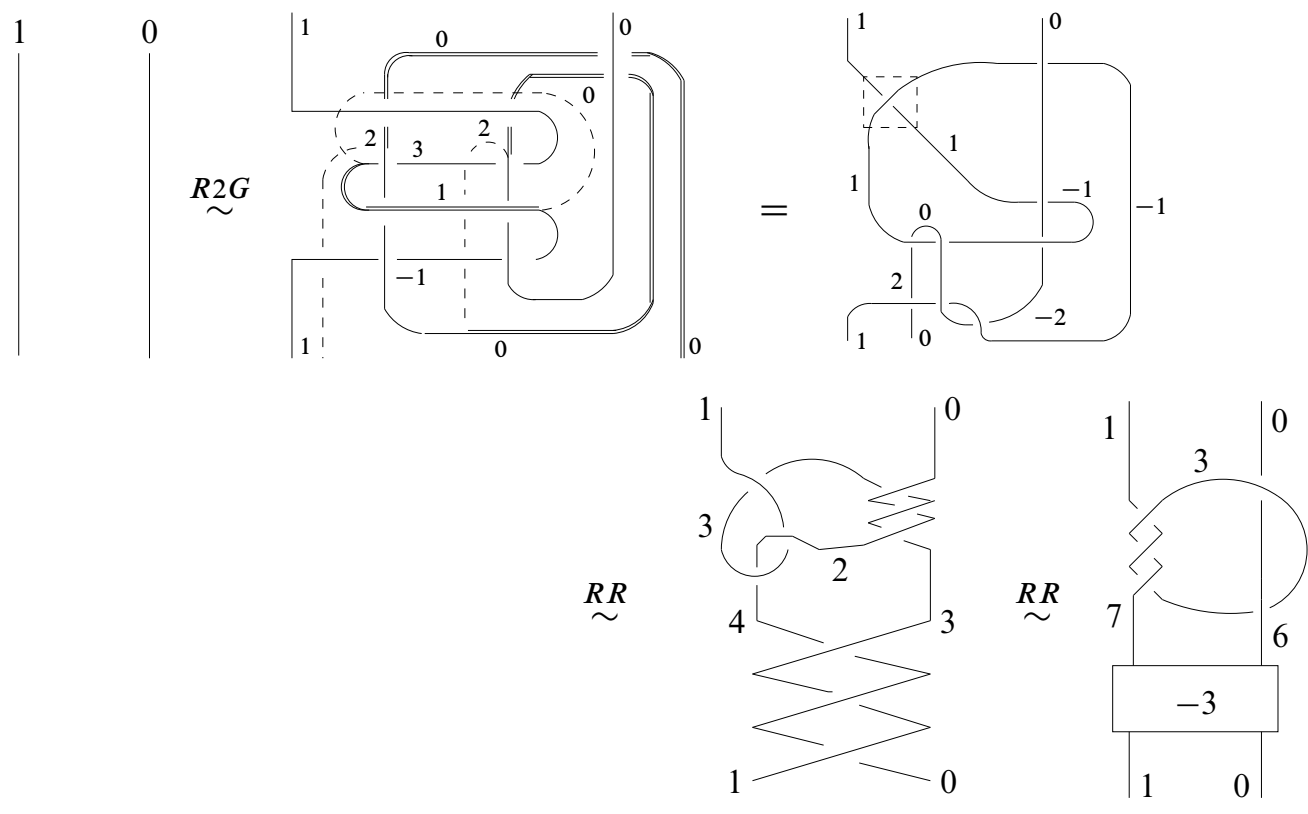

This proves the corollary.

Before we state the next corollary, we define a connect-sum for $p$-coloured knots. Let $\left(K_{1}, \rho_{1}\right)$ and $\left(K_{2}, \rho_{2}\right)$ be two $p$-coloured knots. We equip both with orientations and basepoints denoted $\star_{1}$ and $\star_{2}$ correspondingly. With respect to this information the connect-sum $\left(K_{1}, \rho_{1}\right) \#_{\left(\star_{1}, \star_{2}\right)}\left(K_{2}, \rho_{2}\right)$ is defined by cutting out neighbourhoods of $\star_{1}$ in $K_{1}$ and of $\star_{2}$ in $K_{2}$ and gluing the ends by two parallel overpaths. If the $p$-colouring of the arc containing $\star_{1}$ is different from the $p$-colouring of the arc containing $\star_{2}$, we change the $p$-colouring of $K_{2}$ by a rotation of the set of colours $\mathbb{Z}_{p}$ before gluing. The colouring of the connect-sum is then uniquely induced by the colouring of the connect-summands.

Lemma 4 A connect-sum of $p$-coloured knots is independent of the choices of basepoints and for connect-sums where one of the connect-summands is a $(p, 2)$-torus knot is independent of the orientation of that connect-summand.

Proof Note that an equivalent definition of connect-summing $p$-coloured knots would be to cut out a neighbourhood of $\star_{2}$ to obtain a $p$-coloured tangle $T$, to make $T$ 'very small', and then to glue $T$ into $K_{1}$ in place of a neighbourhood of $\star_{1}$ according to the orientations of $K_{1}$ and $T$, with $p$-colourings as before. 
Let $\star_{1}$ and $\star_{1}^{\prime}$ be basepoints of $\left(K_{1}, \rho_{1}\right)$, and $\star_{2}$ be a basepoint of $\left(K_{2}, \rho_{2}\right)$. Begin with $\left(K_{1}, \rho_{1}\right) \#_{\left(\star_{1}, \star_{2}\right)}\left(K_{2}, \rho_{2}\right)$ and making $T$ 'very small', slide it along $\left(K_{1}, \rho_{1}\right)$ to a neighbourhood of $\star_{1}^{\prime}$. As $T$ moves under an over-crossing arc of $K_{1}$ coloured $c \in \mathbb{Z}_{p}$, its colouring changes by a reflection by $c$ of $\mathbb{Z}_{p}$. We may reverse this reflection (so that we remain with only a rotation on the set of colours) by the following sequence in which a loop is added by a Reidemeister I move, $T$ is pushed through it by ambient isotopy, and the loop is deleted by another Reidemeister I move:

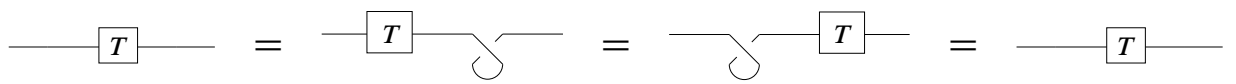

Thus $\left(K_{1}, \rho_{1}\right) \#_{\left(\star_{1}, \star_{2}\right)}\left(K_{2}, \rho_{2}\right)=\left(K_{1}, \rho_{1}\right) \#_{\left(\star_{1}^{\prime}, \star_{2}\right)}\left(K_{2}, \rho_{2}\right)$ and the connect-sum of $p$-coloured knots does not depend on the choice of basepoint.

If $\left(K_{2}, \rho_{2}\right)$ is a $(p, 2)$-torus knot, then $K_{2}$ is invertible. The ambient isotopy from $K_{2}$ to $K_{2}$ with the opposite orientation is given by taking a diagram of $K_{2}$ as below and rotating the plane with respect to which the diagram is taken by $\pi$ (this is the order 2 symmetry of the $(p, 2)$-torus knot- see Kawauchi [7]).

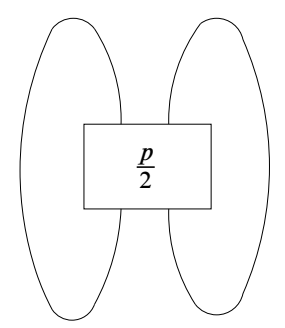

Such an ambient isotopy reflects the set of colours $\mathbb{Z}_{p}$. As before, we may reverse this reflection by sequence (1), giving us the connect-sum of $\left(K_{2}, \rho_{2}\right)$ with the opposite orientation with $\left(K_{1}, \rho_{1}\right)$.

From now on let $\left(K_{1}, \rho_{1}\right) \#\left(K_{2}, \rho_{2}\right)$ denote the connect-sum of $\left(K_{1}, \rho_{1}\right)$ and $\left(K_{2}, \rho_{2}\right)$. When one of the connect summands is a $(p, 2)$-torus knot this is independent of orientation, and in other cases orientations of connect-summands are mentioned explicitly in the text.

If $p=3$ the statement of Corollary 3 simplifies even further, giving us what may be thought of as the key lemma in the proof for the three coloured case.

Corollary 5 For $p=3$, adding 3 twists in a band is equivalent modulo surgery in ker $\rho$ to taking a connect sum with a left-hand trefoil. 
Proof
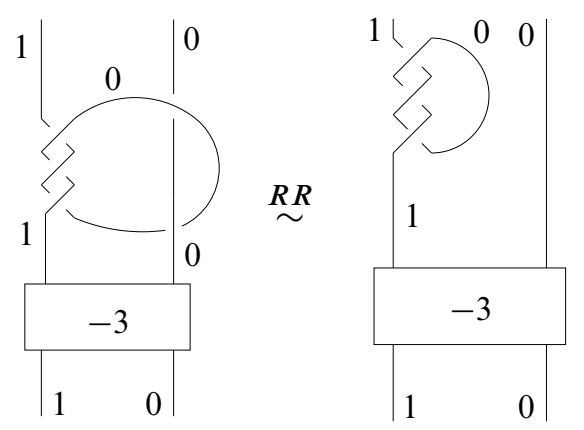

This proves the corollary.

\section{Presenting a $p$-coloured knot as a connect-sum of $p$-col- oured knots of the form $S(m, n)$}

Let $S(m, n)$ (S for Seifert) denote a genus 1 knot with a single negative half-twist between its bands, $m$ full twists in its left band, and $n$ in its right band. Note that by sliding the leftmost band around the disk and reflecting we see that the mirror image of $S(m, n)$ is $S(-m,-n)$. In this section we show that any $p$-coloured knot may be reduced to a connect-sum of $p$-coloured knots of the form $S(m, n)$.

We now state the condition for an $S(m, n)$ knot to be $p$-colourable.

Proposition $6 S(m, n)$ is $p$-colourable if and only if $4 m n \equiv 1 \bmod p$, and if $4 m n \not \equiv 1 \bmod p^{2}$ then this $p$-colouring is unique (up to automorphisms of $D_{2 p}$ ).

Proof The Seifert matrix of $S(m, n)$ is $M=\left(\begin{array}{cc}m & 1 \\ 0 & n\end{array}\right)$, and thus its determinant is $\operatorname{det}\left(M+M^{T}\right)=\left|H_{1}\left(\widehat{C}_{2}(S(m, n))\right)\right|=4 m n-1$, where $\left|H_{1}\left(\widehat{C}_{2}(S(m, n))\right)\right|$ denotes the order of the first homology group of the 2-fold covering of $S^{3}$ branched over $S(m, n)$. If $p$ divides this number but $p^{2}$ does not, then $H_{1}\left(\widehat{C}_{2}(S(m, n))\right)$ contains a single copy of $\mathbb{Z} / p \mathbb{Z}$, and therefore $S(m, n)$ has a $p$-colouring if and only if $4 m n-1 \equiv 0 \bmod p$, and the colouring is unique if $4 m n-1 \not \equiv 0 \bmod p^{2}$. See Fox [4] for details.

All knots are assumed to be given in their band projections (see for instance BurdeZieschang [1, Proposition 8.2]). We remind the reader that this is defined as a projection of a knot $K$ which is represented in $S^{3}$ as the boundary of an orientable surface $F$ in $S^{3}$ with the following properties:

(1) $S=D^{2} \cup B_{1} \cup \cdots \cup B_{2 n}$ where $D^{2}$ and each $B_{j}$ is a disc. 


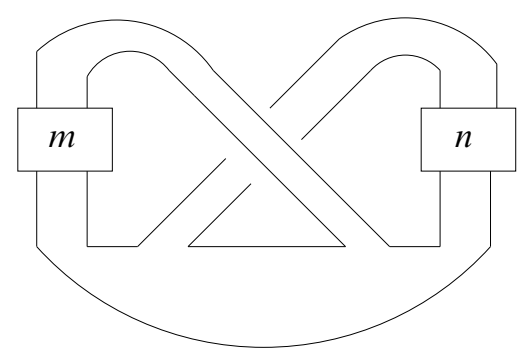

Figure 1: The knot $S(m, n)$

(2) $B_{i} \cap B_{j}=\varnothing$ for $i \neq j, \partial B_{i}=\alpha_{i} \gamma_{i} \beta_{i}\left(\tilde{\gamma}_{i}\right)^{-1}, D^{2} \cap B_{i}=\alpha_{i} \cup \beta_{i}, \partial D^{2}=$ $\alpha_{1} \delta_{1} \beta_{2}^{-1} \delta_{2} \beta_{1}^{-1} \delta_{3} \alpha_{2} \delta_{4} \ldots \alpha_{2 n-1} \delta_{4 n-3} \beta_{2 n}^{-1} \delta_{4 n-2} \beta_{2 n-1}^{-1} \delta_{4 n-1} \alpha_{2 n} \delta_{4 n}$.

In this section, twists in the bands themselves are ignored. We show that for any $p$, the bands of a knot may be unlinked by \pm 1 -framed surgery in ker $\rho$. This implies:

Proposition 7 For any $p$-coloured knot $(K, \rho)$ of genus $n$ there exists a natural number $g$ and integers $m_{1}, m_{2}, \ldots, m_{g}$ and $n_{1}, n_{2}, \ldots, n_{g}$ such that

$$
K \sim S\left(m_{1}, n_{1}\right) \# S\left(m_{2}, n_{2}\right) \# \cdots \# S\left(m_{g}, n_{g}\right)
$$

and each connect-summand has a non-trivial $p$-colouring induced by $\rho$, and the connect-sum is with respect to some orientation of the connect-summands.

The way in which we unlink depends on the colouring of the arcs which border the bands. We thus define the following 'invariant' of bands.

Definition 8 Let $a, b, c, d \in \mathbb{Z}_{p}$ be the colours of the arcs of the band $A$ where it connects to the disc, read from left to right (in the notation above, for $A=\gamma_{i} \cup\left(\tilde{\gamma}_{i}\right)^{-1}$, $a$ is the colour of the arc which contains the point $\gamma_{i} \cap \alpha_{i}, b$ of the arc which contains the point $\tilde{\gamma}_{i} \cap \alpha_{i}, c$ of $\gamma_{i} \cap \beta_{i}$, and $d$ of $\left.\tilde{\gamma}_{i} \cap \beta_{i}\right)$. Let $\underline{a}, \underline{b}, \underline{c}, \underline{d} \in\{0,1,2 \ldots, p-1\}$ represent $a, b, c$ and $d$. We define

$$
|A|:=\min (|\underline{a}-\underline{b}|, p-|\underline{a}-\underline{b}|)=\min (|\underline{d}-\underline{c}|, p-|\underline{c}-\underline{d}|)
$$

the number $|A|$ is independent of twists in $A$ and of crossings of $A$ with other bands. It is called the index of $A$. It corresponds to the image under $\rho$ of a path which loops once around the band.

In the simplest case, bands may be unlinked by surgery along a single component which circles them once. 


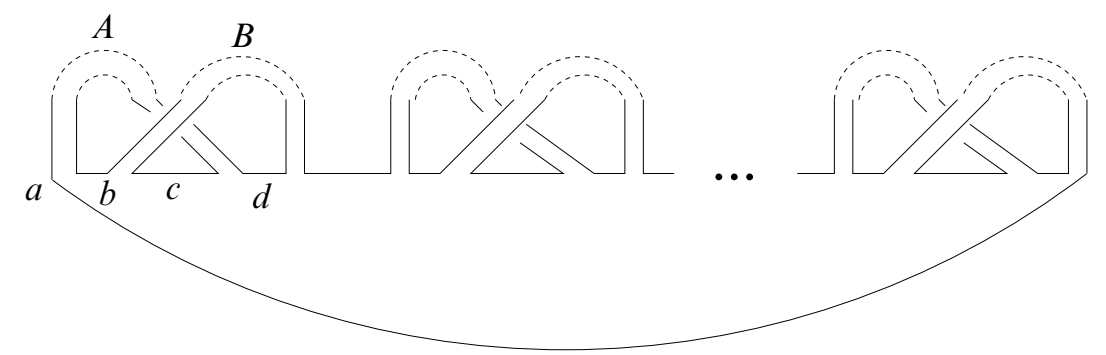

Figure 2: A band projection. Each band may twist and link with any other band.
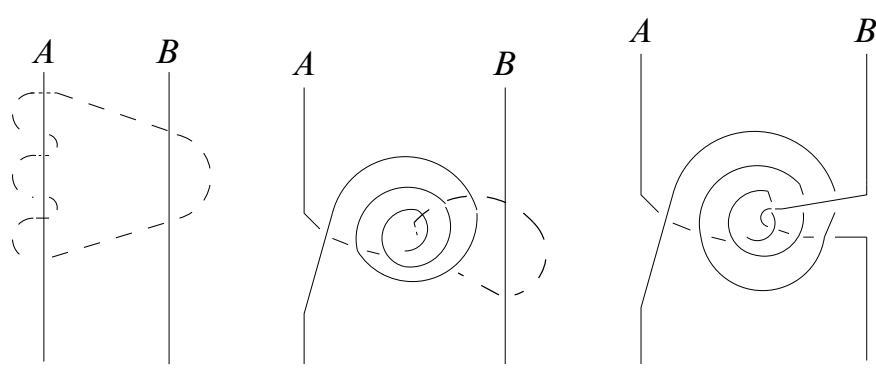

Figure 3: Adding 3 twists between bands $A$ and $B$ for which $3|A|+|B| \equiv 0$ mod $p$. The solid lines represent bands, which we allow to pass through themselves freely by Lemma 9, while the dotted line represents the surgery component.

Lemma 9 If $|A|=|B|$ then $A$ and $B$ may be unlinked in $\operatorname{ker} \rho$.

Proof If $|A|=|B|$, let $C$ be a 1 -framed unknot which circles $A$ and $B$ once. As we may add and subtract a single twist in either band, we may assume that the strands ringed by $C$ are coloured $a, a+r, b$, and $b-r$, and that therefore $C \in \operatorname{ker} \rho$. Surgery along $C$ unlinks the bands.

In the next simplest case, to unlink the bands we require more components and some basic number theory.

Lemma 10 If $|A| \neq|B|$ are both nonzero, then $A$ and $B$ may be unlinked in ker $\rho$.

Proof Pick $a$ and $b$ coprime to $p$ such that $a|A|+b|B| \equiv 0 \bmod p$. Let $C$ be the component in $H_{1}\left(S^{3}-K\right)$ which (from some choice of base-point) circles $a$ times around $A$ and then $b$ times around $B$. The surgery component $C$ is in $\operatorname{ker} \rho$, and the effect of surgery by $C$, by applying the previous lemma (which allows us to pass a 
band through itself) is to add $a b$ twists between the bands $A$ and $B$. The example $a=3$ and $b=1$ is illustrated in Figure 3 .

Because $p$ is prime, we may assume by an automorphism of $D_{2 p}$ that $|A|=a=1$. We have $1+b|B| \equiv 0 \bmod p$ and also $1+(b-p) \bmod p|B| \equiv 0 \bmod p$, hence we may add or subtract $b$ twists and $p-b$ twists between $A$ and $B$. Since $p$ is prime, $b$ and $p-b$ are coprime and therefore there exist integers $m$ and $n$ such that $m b+n(p-b)=1$. So by subtracting $b$ twists between the bands $m$ times and subtracting $p-b$ twists between the bands $n$ times, we may subtract a single twist between $A$ and $B$.

Proof of Proposition 7 Assume first that $|A|=|B|=0$ and that $A$ and $B$ link with some other bands of non-zero index. Assume also that $A$ and $B$ are the leftmost and the second-to-leftmost bands of $K$ (this can be achieved by rotating the band projection of the knot around the disc). The following sequence allows us to reduce the genus of $K$ by surgery in $\operatorname{ker} \rho$.
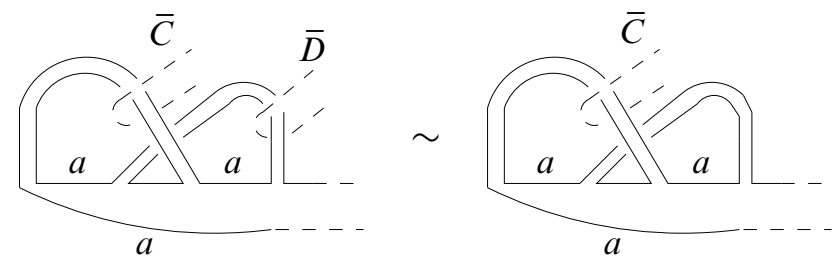

The dotted line denotes any linkage with any other bands, collectively called $\bar{C}$ and $\bar{D}$. To get from the first diagram to the second, notice that conjugation of $a$ by $\bar{C}$ and by $\bar{D}$ gives $a$. Thus the component which loops once around $\bar{D}$ and once around $B$ is in ker $\rho$, and surgery on this component followed by $R R$ to undo the twist we get in $B$ allows us to unlink $B$ and $\bar{D}$ and then to isotopy $\bar{D}$ out of the picture. The resulting diagram represents a $p$-coloured knot of lower genus that the one which we started with, because we may slide $\bar{C}$ out of the picture by ambient isotopy (or alternatively repeat the above surgery argument to unlink $\bar{C}$ from $A$ ) and then eliminate $A$ and $B$ by ambient isotopy.

We are left now only with the case $|A| \neq 0$ but $|B|=0$. In this case we choose a new disc-band presentation for $K$. The procedure we carry out below is equivalent to sliding $A$ over $B$. In the notation at the beginning of the section, we take $A=B_{1}$ and $B=B_{2}$. Choose $D^{\prime 2}, B_{1}^{\prime}, \ldots, B_{2 n}^{\prime}$ such that

(1) $S=D^{\prime 2} \cup B_{1}^{\prime} \cup \cdots \cup B_{2 n}^{\prime}$ where $D^{\prime 2}$ and each $B_{j}^{\prime}$ is a disc, and $B_{i}^{\prime}=B_{i}$ for $i=1$ and for $3 \leq i \leq 2 n$; 

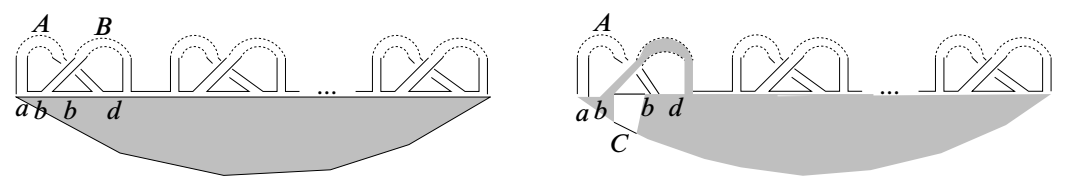

Figure 4: Two choices of cut systems for a Seifert surface when $|B|=0$ but $|A| \neq 0$

(2) $\partial B^{\prime 2}=\alpha_{2}^{\prime} \gamma_{2}^{\prime} \beta_{2}^{\prime}\left(\tilde{\gamma}_{2}^{\prime}\right)^{-1}$ where $\alpha_{2}^{\prime} \subset \delta_{2}, \beta_{2}^{\prime} \subset \delta_{2 n}$, and $\gamma_{2}^{\prime}$ and $\tilde{\gamma}_{2}^{\prime}$ are nonintersecting simple curves in $D^{2}$ connecting the leftmost endpoint of $\alpha_{2}^{\prime}$ with the leftmost endpoint of $\beta_{2}^{\prime}$ and the rightmost endpoint of $\alpha_{2}^{\prime}$ with the rightmost endpoint of $\beta_{2}^{\prime}$ correspondingly.

Note that the above data also uniquely characterizes $D^{\prime 2}$. Let $B_{2}^{\prime}$ be renamed $C$. We get a new disc-band presentation of the Seifert surface, in which both $|A|$ and $|C|$ are nonzero. See Figure 4 (note that $A$ and $B$ need not be in leftmost position).

Repeating for all bands whose index is zero, we obtain a knot whose bands all have non-zero index, reducing the problem to a previously considered case.

That the $K_{i}$ are $p$-colourable follows from examining the $p$-colouring of $K_{i}$ as a connect summand of $K$. Assume that $K_{i}$ has two bands $A$ and $B$ with $A$ to the left of $B$. Assume $A$ has $m$ twists and $B$ has $n$ twists. Then if $a$ is 0 and if $b$ is 1 (notation as in Definition 8) then $c$ is $2 m$ and $d$ is $2 m+1$. Labeling the colours of the corresponding strands of $B$ left to right by $a^{\prime}, b^{\prime}, c^{\prime}, d^{\prime}$ we have $a^{\prime}=2 m, b^{\prime}=1$, and $c^{\prime}=2 m+1$. This forces $d^{\prime}$ to be 0 , proving that $K_{i}$ is indeed $p$-colourable with colouring $\rho_{i}$ induced by $\rho$.

If any of the connect-summands are trivially coloured, they may be untied by the $R R$-move, and thus we may assume that all connect-summands come equipped with a non-trivial $p$-colouring.

\section{Three colours}

In this section we prove the first part of Theorem 1 for $p=3$. We recall that this states that there are at most 3 equivalence classes of 3 -coloured knots modulo surgery in $\operatorname{ker} \rho$, and that these equivalence classes are represented by connect-sums of $n$ left-hand trefoils for $n=1,2,3$. The remaining part of the theorem for $p=3$, that there are at least 3 such equivalence classes, is proved in Section 6. 
Proof of Theorem 1 (first part, $p=3$ case) Let $(K, \rho)$ be a 3 -coloured knot. By Proposition 7 we have

$$
(K, \rho) \sim S\left(m_{1}, n_{1}\right) \# S\left(m_{2}, n_{2}\right) \# \cdots \# S\left(m_{g}, n_{g}\right)
$$

where the 3-colourings of the connect-summands are induced by $\rho$, and the connectsum is with respect to some orientation of the connect-summands. By the $L T$ move (Corollary 5) we may 3-reduce the number of twists in the bands of each connectsummand, allowing us to express $K$ as a connect-sum of 3 -coloured $S(m, n)$ knots with $-1 \leq n, m \leq 1$. By Proposition 6, there are two such 3-colourable knots- the left-hand trefoil $S(1,1)$ which we denote $3_{1}$, and the right-hand trefoil $S(-1,-1)$ which we denote $\overline{3_{1}}-$ and thus $(K, \rho)$ is equivalent to a connect-sum of $3_{1}$ knots and $\overline{3_{1}}$ knots modulo surgery in $\operatorname{ker} \rho$.

By $L T$ on the three half-twists of the trefoil, we have

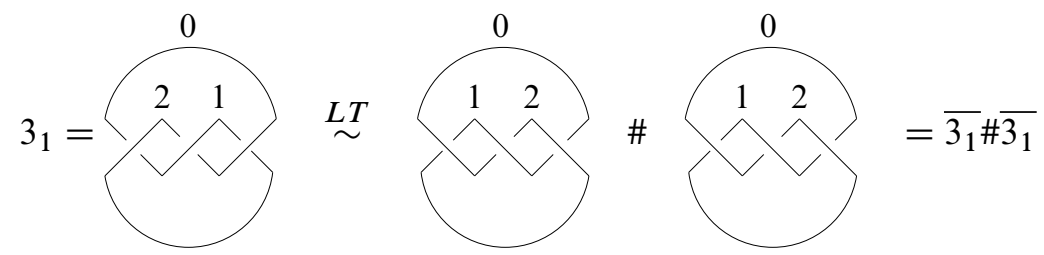

By reflection we also have $\overline{3_{1}} \sim 3_{1} \# 3_{1}$. This shows that $(K, \rho)$ is equivalent to a connect-sum of left-hand trefoils modulo surgery in $\operatorname{ker} \rho$. The number of connectsummands may be 3 -reduced as follows:

$$
\left(3_{1}\right)^{\# 4} \sim\left(\overline{3_{1}}\right)^{\# 2} \sim 3_{1}
$$

Thus $(K, \rho)$ is equivalent to $3_{1}$ or $\left(3_{1}\right)^{\# 2}$ or $\left(3_{1}\right)^{\# 3}$.

\section{Five colours}

In this section we prove the first part of Theorem 1 for $p=5$. This says that there are at most 5 equivalence classes of 5-coloured knots modulo surgery in $\operatorname{ker} \rho$, and that these equivalence classes are represented by connect-sums of $n$ left-hand $5_{1}$ knots for $n=1,2,3,3,4,5$. The remaining part of the theorem for $p=5$, that there are at least 5 such equivalence classes, is proved in Section 6.

We give first the proof, and then fill in the necessary lemmata in the coming sections.

Proof of Theorem 1 (first part, $p=5$ case) Let $(K, \rho)$ be a 5 -coloured knot. By Proposition 7 know that

$$
(K, \rho) \sim S\left(m_{1}, n_{1}\right) \# S\left(m_{2}, n_{2}\right) \# \cdots \# S\left(m_{g}, n_{g}\right)
$$



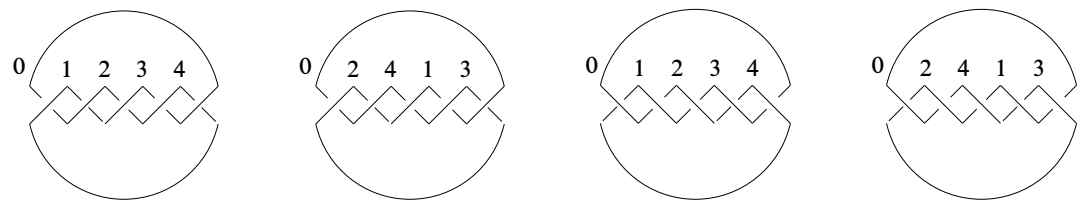

Figure 5: From left to right: $\left(5_{1}, \rho_{1}\right),\left(5_{1}, \rho_{2}\right),\left(\overline{5_{1}}, \overline{\rho_{1}}\right)$, and $\left(\overline{5_{1}}, \overline{\rho_{2}}\right)$

where the 5-colourings of the connect-summands are induced by $\rho$, and the connectsum is with respect to some orientation of the connect-summands.

We may 5-reduce the number of twists in the bands of each $S(m, n)$ connect-summand by the $10-$ move (Proposition 13), allowing us to express $K$ as a connect-sum of $5_{1}$ knots with knots of the form $S(m, n)$ with $-2 \leq n, m \leq 2$ whose unique 5-colouring is induced by $\rho$. A list of such knots is derived from Proposition 6- these are the knots $S(2,2)$ and $S(-1,1)$. These knots are each reduced to a connect-sum of $5_{1}$ knots by Proposition 14. We thus obtain:

$$
(K, \rho) \sim\left(5_{1}, \rho_{1}\right)^{\# g_{1}} \#\left(5_{1}, \rho_{2}\right)^{\# g_{2}} \#\left(\overline{5_{1}}, \overline{\rho_{1}}\right)^{\# g_{3}} \#\left(\overline{5_{1}}, \overline{\rho_{2}}\right)^{\# g_{4}}
$$

where $\left(5_{1}, \rho_{1}\right),\left(5_{1}, \rho_{2}\right),\left(\overline{5_{1}}, \overline{\rho_{1}}\right)$, and $\left(\overline{5_{1}}, \overline{\rho_{2}}\right)$ denote the $5_{1}$ knots with different handedness and colourings which are not ambient isotopic. See Figure 5.

The theorem now follows from Proposition 16, which tells us that

$$
(K, \rho) \sim\left(5_{1}, \rho_{1}\right)^{\# n} \quad \text { for some } n=1,2,3,4,5 \text {. }
$$

The key steps of the proof are thus:

- To reduce the number of twists in the bands via the 10 -move.

- To reduce $S(m, n)$ to a connect-sum of $5_{1}$ knots for $-2 \leq m, n \leq 2$.

- To reduce a connect-sum of 51 knots to a connect-sum of between one and five $\left(5_{1}, \rho_{1}\right)$ knots.

The first stage is carried out in Section 5.2, the second in Section 5.3, and the third in Section 5.4. Section 5.1 is concerned with preliminary lemmata for use in these sections.

\subsection{Preliminary lemmata}

In the present section we collect together several preliminary lemmata whose main purpose is to relate 5-coloured $55_{1}$ knots to knots and tangles which are technically simpler to compute with.

Algebraic 83 Geometric Topology, Volume 6 (2006) 
We begin with some notation which is used only in this section. We work in the category of $(2,2)$-tangles rotated by $\frac{\pi}{2}$, so that the composition of two tangles is given by stacking from left to right

$$
T_{1} T_{2}:=\begin{array}{|l|l|}
T_{1} & T_{2} \\
\hline
\end{array}
$$

Let $T$ be a 5-coloured $(2,2)$-tangle. For $a$ the 5-colouring of the left endpoint of the top strand of $T$ and $b$ the 5-colouring of the left endpoint of the top strand of $T$, let $\underline{a-b} \in\{-2,-1,0,1,2\}$ represent $a-b$. The absolute value of the difference $d=|\underline{a-b}|$ is identical to the absolute value of the difference of the 5-colourings of the right endpoints, defined the same way.

We now define several 'basic' tangles. Let $\mathbb{1}$ denote the trivial $(2,2)$-tangle and

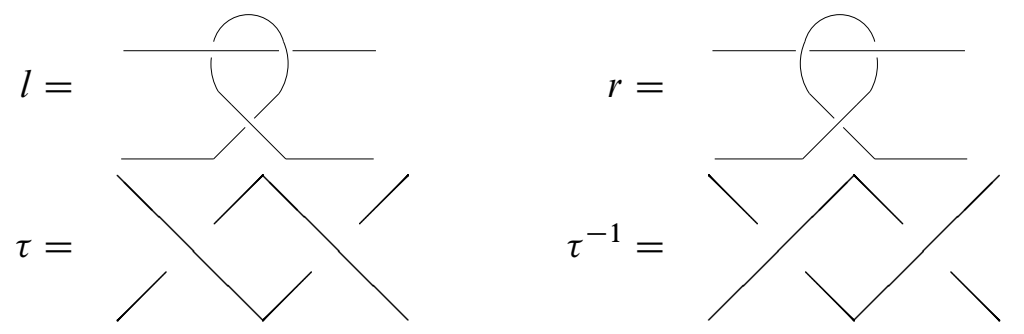

Let $\left(5_{1}, \rho_{0}\right)$ and $\left(\overline{5_{1}}, \overline{\rho_{0}}\right)$ denote the trivially coloured left-hand and right-hand $5_{1}-$ knots correspondingly.

We prove a number of equivalences between the tangles defined above. Define a connect-sum of a $p$-coloured knot with a $p$-coloured $(2,2)$-tangle to be the connectsum of that knot with the bottom strand of the tangle (this is well-defined by Lemma $4)$.

Lemma 11 Let $d=|\underline{a-b}|$ denote the absolute value of the difference between the 5 -colouring of the upper strand and the lower strand as before. Then we have:

(1) $l^{2} \sim r \cdot \tau$ and $r^{2} \sim l \cdot \tau^{-1}$.

(2) $l^{2} \cdot \tau \sim \mathbb{1} \#\left(5_{1}, \rho_{d}\right)$ and $r^{2} \cdot \tau^{-1} \sim \mathbb{1} \#\left(\overline{5_{1}}, \overline{\rho_{d}}\right)$.

Proof Part (1)

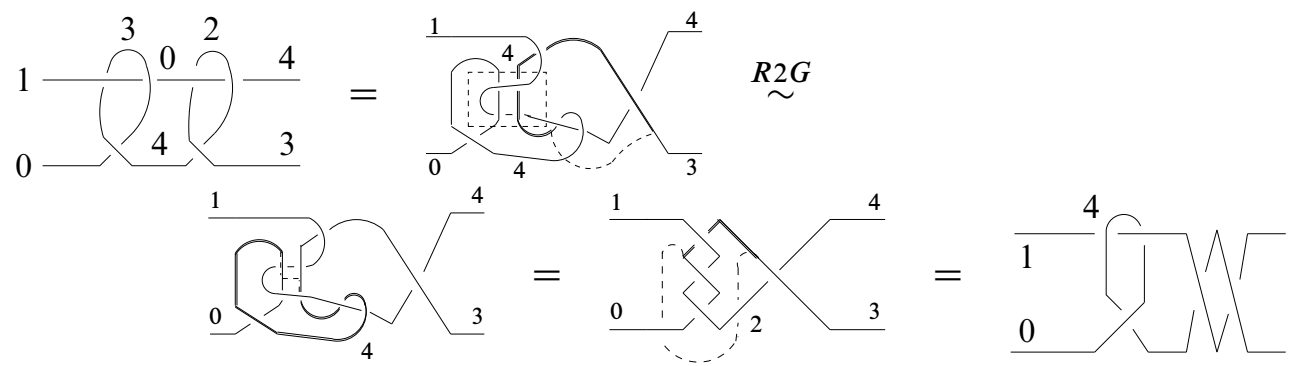

Algebraic 83 Geometric Topology, Volume 6 (2006) 
To get from the second diagram to the first diagram, from the fourth diagram to the fifth diagram, and from the fifth diagram to the final diagram, isotopy the thick line-segment to the position specified by the dotted line. To get from the second diagram to the third, use $R 2 G$ inside the dotted box on the arc coloured 0 through the two arcs coloured 3

The $d=0$ case is trivial. For $d=2$ instead of $d=1$, in the same way we obtain

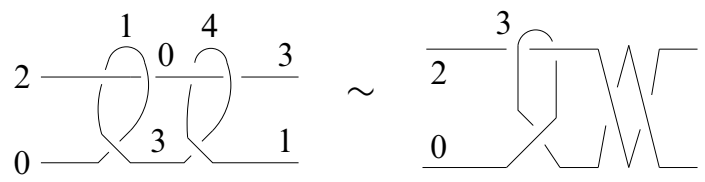

The proof of $r^{2} \sim l \cdot \tau^{-1}$ is the same up to reflection.

Part (2)

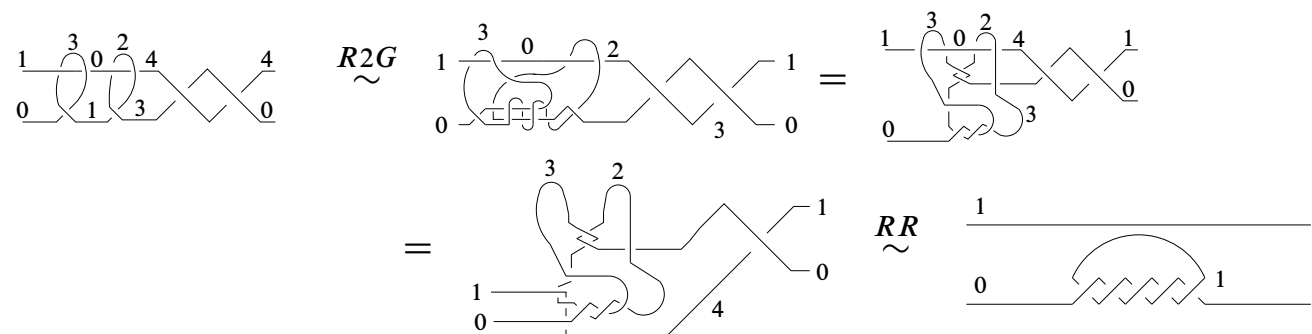

To get from the first diagram to the second diagram, use the $R 2 G$ move on the arc coloured 4 through the two arcs coloured 3.

The $d=0$ case is trivial. For $d=2$ instead of $d=1$, in the same way we obtain

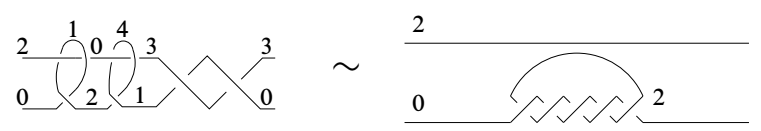

The proof of $r^{2} \cdot \tau^{-1} \sim \mathbb{1} \#\left(\overline{5_{1}}, \overline{\rho_{d}}\right)$ is the same up to reflection.

This implies:

$$
\mathbb{1} \#\left(5_{1}, \rho_{d}\right)^{2} \sim l^{4} \cdot \tau^{2} \sim r^{2} \cdot \tau^{4}=r^{2} \cdot \tau^{-1} \cdot \tau^{5} \sim \tau^{5} \#\left(\overline{5_{1}}, \overline{\rho_{d}}\right)
$$

\subsection{The 10-move}

By Equation (2) on the five half-twists of the (5,2)-torus knot, we have

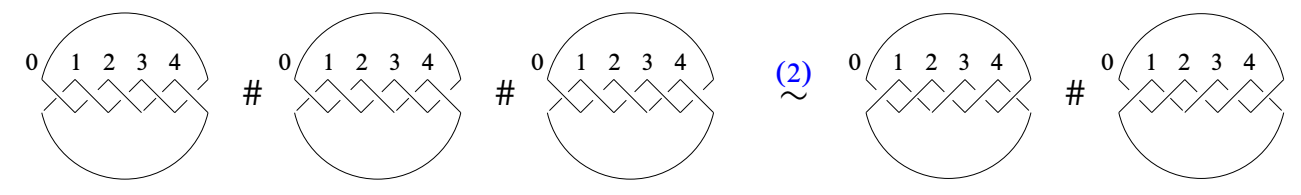


Repeating for all different colourings of the 51 knot we obtain:

$$
\begin{aligned}
& \left(5_{1}, \rho_{1}\right)^{\# 3} \sim\left(\overline{5_{1}}, \overline{\rho_{1}}\right)^{\# 2} \\
& \left(5_{1}, \rho_{2}\right)^{\# 3} \sim\left(\overline{5_{1}}, \overline{\rho_{2}}\right)^{\# 2} \\
& \left(\overline{5_{1}}, \overline{\rho_{1}}\right)^{\# 3} \sim\left(5_{1}, \rho_{1}\right)^{\# 2} \\
& \left(\overline{5_{1}}, \overline{\rho_{2}}\right)^{\# 3} \sim\left(5_{1}, \rho_{2}\right)^{\# 2}
\end{aligned}
$$

where the exponent is with respect to the connect-sum operation.

Lemma 12 Any 5-coloured knot $K$ is equivalent modulo surgery in ker $\rho$ to itself connect-summed with five $5_{1}$ knots of any handedness and any 5-colouring.

Proof Let $\left(K_{0}, \rho\right)$ be a 5 -coloured knot in $S^{3}$, and let $B$ be a ball in $S^{3}$ such that $T:=B \cap K_{0}$ is the trivial $(2,2)$-tangle $\mathbb{1}$ (two unknotted untangled parallel strands), where the colouring of the upper arc is 1 and the colouring of the lower arc is 0 .

$T$ is the product of two trivial $(2,2)-$ tangles $T_{0}$ and $T_{1}$. We first add and subtract $\frac{-3}{2}$ twists from $T_{0}$ by a series of Reidemeister $R I I$ moves, and then we extract a copy of $l$ from these twists by

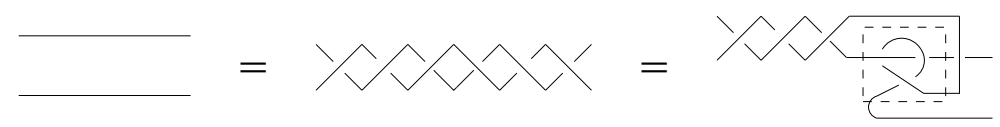

By Lemma 11 this allows us to extract a copy of $\left(5_{1}, \rho_{1}\right)$ from the modified $T_{0}$. We repeat this procedure six times. Then by repeated use of Equations (3) and (4) we have:

$$
\left(5_{1}, \rho_{1}\right)^{\# 6} \sim\left(\overline{5_{1}}, \overline{\rho_{1}}\right)^{\# 6} \#\left(5_{1}, \rho_{1}\right)^{\# 2} \sim\left(5_{1}, \rho_{1}\right)^{\# 11}
$$

By isotopy, we take five copies of $\left(5_{1}, \rho_{1}\right)$ into $T_{1}$ (making it isotopic to a copy of $\left.\left(5_{1}, \rho_{1}\right)^{\# 5}\right)$, while returning the remaining six copies of $\left(5_{1}, \rho_{1}\right)$ to their initial positions in the modified $T_{0}$. Reversing the process on what was $T_{0}$, we may make it once again into a trivial $(2,2)$-tangle. Since we could have chosen $T$ to have any colouring of its upper and lower arcs (by different choices of $B$ ), we may determine the colouring of the $5_{1}$ knots which we obtain as connect-summands.

Reminder Two coloured knots are said to be $2 p$-move equivalent if they are related by a sequence of $2 p$-moves:

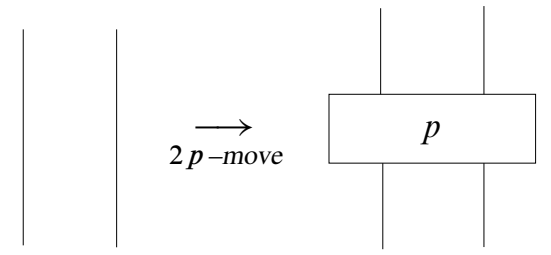

Algebraic 83 Geometric Topology, Volume 6 (2006) 
Proposition 13 Let $K_{0}$ and $K_{1}$ be 10-move equivalent 5-coloured knots. Then $K_{0} \sim K_{1} \#\left(K^{\prime}\right)^{\# 2}$ where $K^{\prime}=\left(5_{1}, \rho_{1}\right)$ or $\left(5_{1}, \rho_{2}\right)$ or $\left(\overline{5_{1}}, \overline{\rho_{1}}\right)$ or $\left(\overline{5_{1}}, \overline{\rho_{2}}\right)$.

Proof We may assume that $K_{1}$ is obtained from $K_{0}$ by a single $10-$ move, by adding ten half twists in two parallel strands of $K_{0}$. By Lemma 12, $K_{0} \sim K_{0} \#\left(5_{1}, \rho_{1}\right)^{\# 5}$ (the proof is the same for different colouring and handedness of the $\left.5_{1}-\mathrm{knot}\right)$. We now look at a trivial $(2,2)$-tangle $\mathbb{1}$ in $K_{0}$ connect-summed with $\left(5_{1}, \rho_{1}\right)^{\# 2}$. By Equation (2) this is equivalent modulo surgery in $\operatorname{ker} \rho$ to $\tau^{5} \#\left(\overline{5_{1}}, \overline{\rho_{1}}\right)$. So we have converted $K_{0}$ to $K_{1} \#\left(5_{1}, \rho_{1}\right)^{\# 3} \#\left(\overline{5_{1}}, \overline{\rho_{1}}\right)$ by \pm 1 -framed surgeries in ker $\rho$. By Equations (3)-(6) we have

$$
\left(5_{1}, \rho_{1}\right)^{\# 3} \#\left(\overline{5_{1}}, \overline{\rho_{1}}\right) \sim\left(\overline{5_{1}}, \overline{\rho_{1}}\right)^{\# 3} \sim\left(5_{1}, \rho_{1}\right)^{\# 2}
$$

which proves the proposition.

\subsection{Reducing $S(m, n)$ to $5_{1}$}

In the present section we prove equivalences between the $5_{1}$ knot and 5-coloured knots of the form $S(m, n)$ with $-2 \leq m, n \leq 2$ (see Figure 1). By Proposition 6 there are two such knots up to reflection- $S(2,2)$, and $S(-1,1)$ - and the 5-colouring of each of them is unique (up to automorphism of $D_{2 p}$ ).

Proposition 14 The 5-coloured knots $S(2,2), S(-1,1)$, and their mirror images are all equivalent to connect-sums of $5_{1}$ knots modulo surgery in $\operatorname{ker} \rho$.

Proof $S(-1,1) \sim 5_{1}$ :
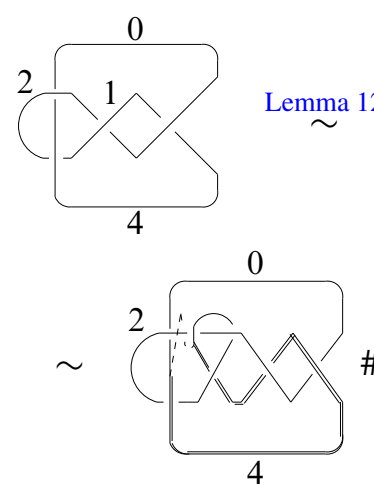

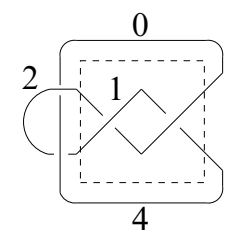

\#4

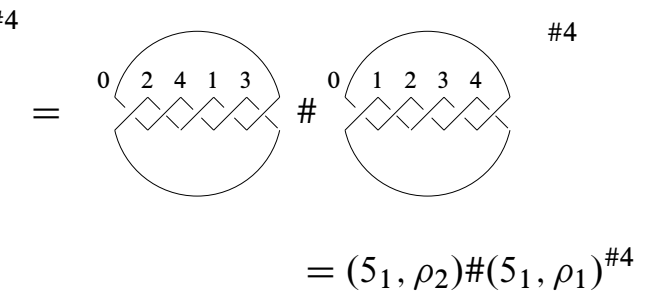

where the second equivalence follows from Lemma 11 by considering the copy of $\tau^{-1}$ in $S(-1,1)$ in the dotted box connect-summed with one of the copies of $\left(5_{1}, \rho_{1}\right)$. We then have

$$
\tau^{-1} \#\left(5_{1}, \rho_{1}\right)=\tau^{-1} \cdot \tau \cdot l^{2}=r \cdot \tau
$$


$S(2,2) \sim 5_{1}:$
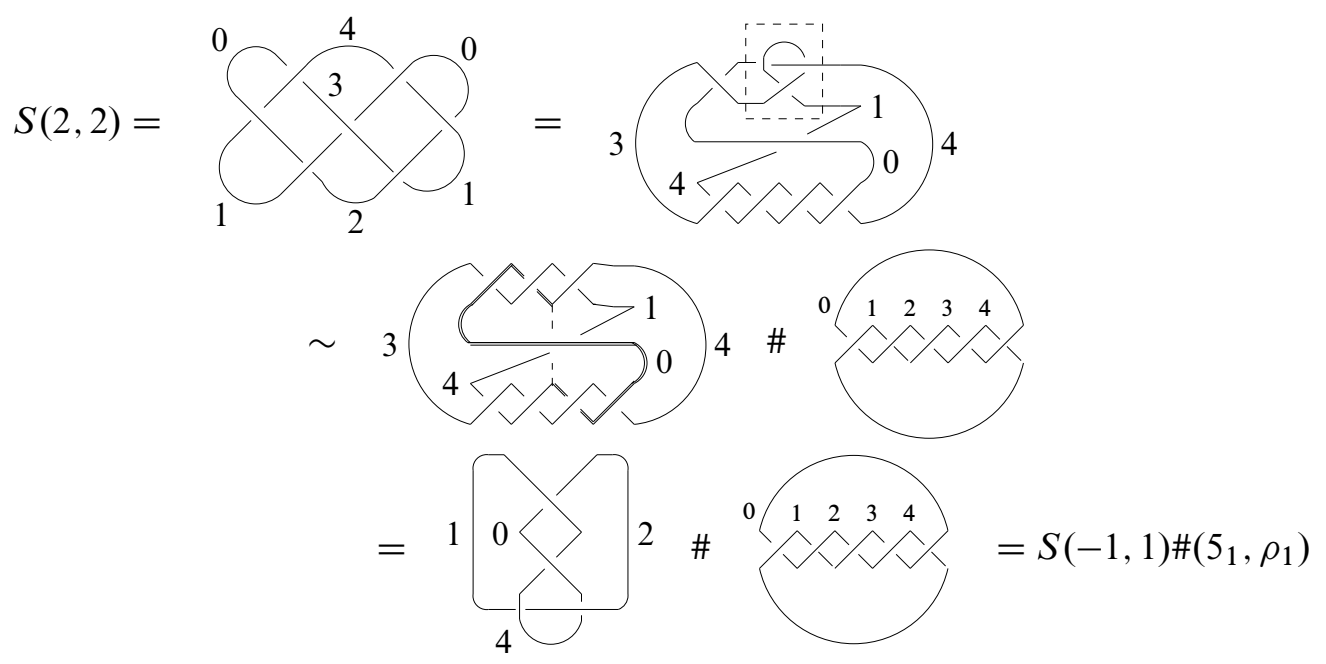

To get from the second diagram to the third, use Lemma 11 to deduce

$$
r=r \cdot \tau^{2} \cdot \tau^{-2}=l^{2} \cdot \tau \cdot \tau^{-2}=\left(5_{1}, \rho_{a}\right) \# \tau^{-2}
$$

where the copy of $r$ in the second diagram is drawn inside a dotted box. In the final stage we have reduced to the previous case.

\subsection{Reducing the number of connect summands}

Let

$$
(K, \rho)=\left(5_{1}, \rho_{1}\right)^{\# g_{1}} \#\left(5_{1}, \rho_{2}\right)^{\# g_{2}} \#\left(\overline{5_{1}}, \overline{\rho_{1}}\right)^{\# g_{3}} \#\left(\overline{5_{1}}, \overline{\rho_{2}}\right)^{\# g_{4}}
$$

be a connect-sum of $5_{1}$-knots. The purpose of this section is to prove that

$$
(K, \rho) \sim\left(5_{1}, \rho_{1}\right)^{\# n} \quad \text { for some } n=1,2,3,4,5 .
$$

We achieve this by finding relations between connect-sums of 51 knots with different colouring and handedness.

\section{Lemma 15}

$$
\begin{aligned}
& \left(5_{1}, \rho_{1}\right) \#\left(\overline{5_{1}}, \overline{\rho_{1}}\right)^{2} \sim\left(5_{1}, \rho_{2}\right) \\
& \left(\overline{5_{1}}, \overline{\rho_{1}}\right) \#\left(5_{1}, \rho_{1}\right)^{2} \sim\left(\overline{5_{1}}, \overline{\rho_{2}}\right) \\
& \left(5_{1}, \rho_{2}\right) \#\left(\overline{5_{1}}, \overline{\rho_{2}}\right)^{2} \sim\left(\left(5_{1}, \rho_{1}\right)\right. \\
& \left(\overline{5_{1}}, \overline{\rho_{2}}\right) \#\left(5_{1}, \rho_{2}\right)^{2} \sim\left(\overline{5_{1}}, \overline{\rho_{1}}\right)
\end{aligned}
$$


Proof We prove Equation (7). The proofs of the other equations in the lemma follow by reflection and automorphism of $D_{10}$. Consider:

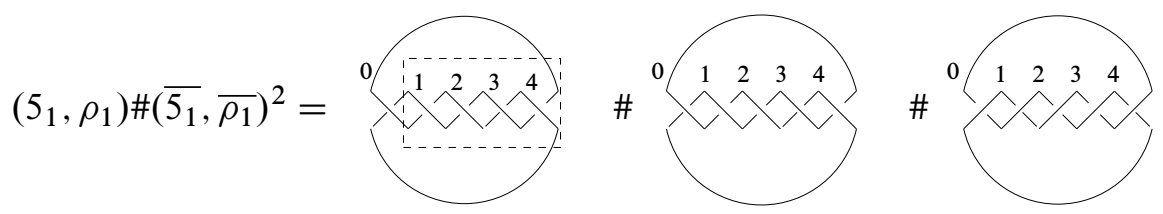

Consider two of the twists (four of the half-twists) in one of the copies of $\left(\overline{5_{1}}, \overline{\rho_{1}}\right)$ as the $(2,2)$-tangle $\tau^{2}$, and the remaining $\left(\overline{5_{1}}, \overline{\rho_{1}}\right)$ and $\left(5_{1}, \rho_{1}\right)$ as being connect-summed to this $(2,2)$-tangle. We have:

$$
\tau^{2} \#\left(\overline{5_{1}}, \overline{\rho_{1}}\right) \sim r^{2} \cdot \tau^{-1} \cdot \tau^{2}=r^{2} \cdot \tau \sim l \cdot \tau^{-1} \cdot \tau=l
$$

The equation above implies that:

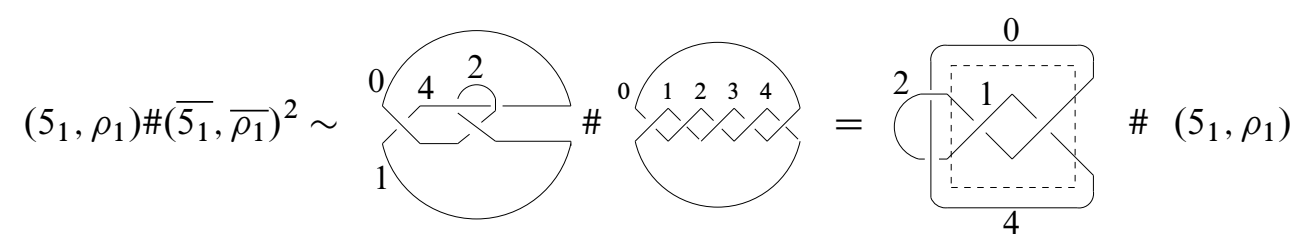

where by the last three steps of the reduction of $S(-1,1)$ to a connect-sum of $5_{1}$ knots in the proof of Proposition 14, the knot at the end of this sequence is equivalent to $\left(5_{2}, \rho_{2}\right)$ modulo surgery in $\operatorname{ker} \rho$.

\section{Proposition 16}

$$
(K, \rho)=\left(5_{1}, \rho_{1}\right)^{\# g_{1}} \#\left(5_{1}, \rho_{2}\right)^{\# g_{2}} \#\left(\overline{5_{1}}, \overline{\rho_{1}}\right)^{\# g_{3}} \#\left(\overline{5_{1}}, \overline{\rho_{2}}\right)^{\# g_{4}} \sim\left(5_{1}, \rho_{1}\right)^{n}
$$

for $n=1,2,3,4,5$.

Proof Using Lemma 15 and Equations (3)- (6) we have:

$$
\begin{aligned}
\left(5_{1}, \rho_{2}\right) & \sim\left(5_{1}, \rho_{1}\right) \#\left(\overline{5_{1}}, \overline{\rho_{1}}\right)^{\# 2} \sim\left(5_{1}, \rho_{1}\right)^{\# 4} \\
\left(\overline{5_{1}}, \overline{\rho_{1}}\right) & \sim\left(5_{1}, \rho_{2}\right)^{\# 2} \#\left(\overline{5_{1}}, \overline{\rho_{2}}\right) \sim\left(\left(5_{1}, \rho_{1}\right)^{\# 2} \#\left(\overline{5_{1}}, \overline{\rho_{1}}\right)\right) \#\left(\left(5_{1}, \rho_{1}\right) \#\left(\overline{5_{1}}, \overline{\rho_{1}}\right)^{\# 2}\right)^{\# 2} \\
& =\left(5_{1}, \rho_{1}\right)^{\# 4} \#\left(\overline{5_{1}}, \overline{\rho_{1}}\right)^{\# 5} \sim\left(5_{1}, \rho_{1}\right)^{\# 9} \\
\left(\overline{5_{1}}, \overline{\rho_{2}}\right) & \sim\left(5_{1}, \rho_{1}\right)^{\# 2} \#\left(\overline{5_{1}}, \overline{\rho_{1}}\right) \sim\left(5_{1}, \rho_{1}\right)^{\# 11}
\end{aligned}
$$

which gives

$$
(K, \rho) \sim\left(5_{1}, \rho_{1}\right)^{\# n}
$$

for some positive integer $n$. We may take $n$ to be between one and five since:

Algebraic 83 Geometric Topology, Volume 6 (2006) 


$$
\begin{aligned}
\left(5_{1}, \rho_{1}\right) \sim\left(5_{1}, \rho_{2}\right) \#\left(\overline{5_{1}}, \overline{\rho_{2}}\right)^{\# 2} \sim\left(\left(5_{1}, \rho_{1}\right)^{\# 2} \#\left(\overline{5_{1}}, \overline{\rho_{1}}\right)\right)^{\# 2} \#\left(\left(5_{1}, \rho_{1}\right) \#\left(\overline{5_{1}}, \overline{\rho_{1}}\right)^{\# 2}\right) \\
=\left(5_{1}, \rho_{1}\right)^{\# 5} \#\left(\overline{5_{1}}, \overline{\rho_{1}}\right)^{\# 4} \sim\left(5_{1}, \rho_{1}\right)^{\# 2} \#\left(\overline{5_{1}}, \overline{\rho_{1}}\right)^{\# 6} \sim\left(5_{1}, \rho_{1}\right)^{\# 6}
\end{aligned}
$$

which completes the proof.

\section{An invariant for $p$-coloured knots}

In Section 4 and in Section 5, it was shown that for $p=3$ or $p=5$, any $p$-coloured knot $(K, \rho)$ can be reduced to a connect-sum of $n$ left-hand $(p, 2)$-torus knots with a given colouring for some $1 \leq n \leq p$ by a series of surgeries in $\operatorname{ker} \rho$. In the present section we complete the proof of Theorem 1 by showing that this result cannot be improved. Explicitly, for $p$ an odd prime (in particular for $p \in\{3,5\}$ ) and for $r_{1} \neq r_{2} \bmod p$ the connect-sum of $r_{1}$ left-hand $(p, 2)$-torus knots with a given colouring is not equivalent modulo surgery in $\operatorname{ker} \rho$ to the connect-sum of $r_{2}$ left-hand $(p, 2)$-torus knots with the same colouring. We prove this by finding a $\mathbb{Z} / p \mathbb{Z}$-valued invariant for all $p$-coloured knots, invariant under surgery in $\operatorname{ker} \rho$, which takes different values on each of these knots. Our reference for algebraic topology is Spanier [12].

We begin by recalling Cappell and Shaneson's notion of a $\bmod p$ characteristic knot [2;3]. Recall the presentation $D_{2 p}:=\left\{t, s \mid t^{2}=s^{p}=1, t s t=s^{p-1}\right\}$ for the dihedral group of order $2 p$. Let $(K, \rho)$ be a $p$-coloured knot with Seifert surface $F$ and Seifert matrix $M$ with respect to a basis $x_{1}, x_{2}, \ldots, x_{2 n}$ of $H_{1}(F)$ with orientations induced by the orientation of $F$. Let $\left(\xi_{1}, \xi_{2}, \ldots, \xi_{2 n}\right)$ be a basis for $H_{1}\left(S^{3}-F\right)$ oriented such that $\operatorname{Link}\left(\xi_{i}, x_{j}\right)=\delta_{i j}$. Then there exists a link $\operatorname{ch}(K, \rho) \subset F$ called a $\bmod p$ characteristic link such that

$$
\rho\left(\xi_{i}\right)=s^{\operatorname{Link}\left(\operatorname{ch}(K, \rho), \xi_{i}\right)}
$$

for all $1 \leq i \leq 2 n$. Recall also that $\rho(\tau)=t$ where $\tau$ denotes the generator of the infinite cyclic covering group. In fact $\operatorname{ch}(K, \rho)$ may be chosen to be a knot, but we do not require this fact here.

Let $\widehat{C}_{2}$ denote the 2 -fold covering of $S^{3}$ branched over $K$, and let $p r$ denote the covering projection. The $p$-colouring $\rho: \pi_{1}\left(S^{3}-K\right) \rightarrow D_{2 p}$ restricts in the doublecovering to a map $\rho /: H_{1}\left(\widehat{C}_{2} ; \mathbb{Z}\right) \rightarrow \mathbb{Z} / p \mathbb{Z}$ which corresponds to a cohomology class $a \in H^{1}\left(\widehat{C}_{2} ; \mathbb{Z} / p \mathbb{Z}\right)$ by the universal-coefficient theorem for cohomology.

To simplify notation, we define $\alpha:=p r^{-1} \operatorname{ch}(K, \rho)$. Since $\xi_{i}$ is in the complement of the Seifert surface, its pre-image in $\widehat{C}_{2}$ is two disjoint circles, while the mod $p$ characteristic link is contained in $F$ and its lift therefore has only one component. Let 
$\left[\mathrm{pr}^{-1} \xi_{i}\right] \in H_{1}\left(\widehat{C}_{2} ; \mathbb{Z}\right)$ and $[\alpha] \in H_{1}\left(\widehat{C}_{2} ; \mathbb{Z}\right)$ denote the homology classes represented by $p r^{-1} \xi_{i}$ and $\alpha$ correspondingly. Equation (11) tells us that

$$
\begin{aligned}
& a \frown\left[p r^{-1} \xi_{i}\right]=\left\langle a,\left[p r^{-1} \xi_{i}\right]\right\rangle=s^{\operatorname{Link}\left(\alpha, p r^{-1} \xi_{i}\right)}= \\
& \left\langle D \partial^{-1}[\alpha],\left[p r^{-1} \xi_{i}\right]\right\rangle \bmod p=D \partial^{-1}[\alpha] \bmod p \frown\left[p r^{-1} \xi_{i}\right]
\end{aligned}
$$

for all $1 \leq i \leq 2 n$, where $D$ denotes the Poincaré duality isomorphism, and $\partial^{-1}[\alpha]$ denotes the pre-image of $[\alpha]$ under the Bockstein homomorphism

$$
\cdots \longrightarrow H_{2}\left(\widehat{C}_{2} ; \mathbb{Z}\right) \longrightarrow H_{2}\left(\widehat{C}_{2} ; \mathbb{Z}\right) \longrightarrow H_{2}\left(\widehat{C}_{2} ; \mathbb{Z} / p \mathbb{Z}\right) \stackrel{\partial}{\longrightarrow} H_{1}\left(\widehat{C}_{2} ; \mathbb{Z}\right) \longrightarrow \cdots
$$

where the above long exact sequence is associated to the short exact sequence

$$
0 \longrightarrow \mathbb{Z} \stackrel{-\times p}{\longrightarrow} \mathbb{Z} \stackrel{\text { projection }}{\longrightarrow} \mathbb{Z} / p \mathbb{Z} \longrightarrow 0
$$

Thus

$$
a=D \partial^{-1}[\alpha] \bmod p
$$

The short exact sequence

$$
0 \longrightarrow \mathbb{Z} \stackrel{-\times p}{\longrightarrow} \mathbb{Z} \stackrel{\text { projection }}{\longrightarrow} \mathbb{Z} / p \mathbb{Z} \longrightarrow 0
$$

also gives rise to the long exact sequence on cohomology

$$
\cdots \longrightarrow H^{1}\left(\hat{C}_{2} ; \mathbb{Z}\right) \longrightarrow H^{1}\left(\hat{C}_{2} ; \mathbb{Z}\right) \longrightarrow H^{1}\left(\hat{C}_{2} ; \mathbb{Z} / p \mathbb{Z}\right) \stackrel{\partial^{*}}{\longrightarrow} H^{2}\left(\widehat{C}_{2} ; \mathbb{Z}\right) \longrightarrow \cdots
$$

where $\partial^{*}$ is the Bockstein homomorphism on cohomology. We now define the coloured untying invariant of $(K, \rho)$ as

$$
\operatorname{cu}(K, \rho):=\partial^{*} a \smile a \in H^{3}\left(\widehat{C}_{2} ; \mathbb{Z} / p \mathbb{Z}\right) \cong \mathbb{Z} / p \mathbb{Z}
$$

This gives a non-trivial $\mathbb{Z} / p \mathbb{Z}$-valued invariant of $p$-coloured knots.

Proposition 17 The coloured untying invariant is invariant under \pm 1 -framed surgery in $\operatorname{ker} \rho$.

Proof By Poincaré duality the dual of $\mathrm{cu}(K, \rho)$ is equal to the algebraic intersection number of $D(a)$ with $\partial D(a)$.

Let $L$ be a loop in $\operatorname{ker} \rho$. Since both components in the pre-image of $L$ in $\widehat{C}_{2}$ vanish as elements of $H_{1}\left(\widehat{C}_{2} ; \mathbb{Z} / p \mathbb{Z}\right)$, these components may be chosen to be disjoint with $D(a)$. Thus performing surgery by $L$ does not change $\mathrm{cu}(K, \rho)$.

The next lemma gives a way to calculate the coloured untying invariant. 
Lemma 18 Let $v:=\left(v_{1}, v_{2}, \ldots, v_{2 n}\right)^{T} \in \mathbb{Z} / p \mathbb{Z}^{2 n}$ be a column vector such that $v_{i}=a \frown\left[p^{-1} \xi_{i}\right]$ for all $1 \leq i \leq 2 n$. Then

$$
\operatorname{cu}(K, \rho)=\frac{2\left(v^{T} \cdot M \cdot v\right)}{p} \bmod p
$$

Proof Notice first that

$$
\frac{2\left(v^{T} \cdot M \cdot v\right)}{p} \bmod p=\frac{v^{T} \cdot\left(M+M^{T}\right) \cdot v}{p} \bmod p
$$

where $v^{T} \cdot\left(M+M^{T}\right) \cdot v$ equals the linking number of $\alpha$ with itself in $\hat{C}_{2}$. By definition we have

$$
\begin{aligned}
& \frac{1}{p} \operatorname{Link}(\alpha, \alpha) \bmod p=\frac{1}{p}\left\langle D \partial^{-1}[\alpha] \bmod p,[\alpha]\right\rangle= \\
& \frac{1}{p} D \partial^{-1}[\alpha] \bmod p \frown[\alpha]=\frac{1}{p}(a \frown[\alpha])=a \frown \partial D(a)= \\
& D\left(a \smile \partial^{*} a\right)=D(\operatorname{cu}(K, \rho))=\operatorname{cu}(K, \rho)
\end{aligned}
$$

which completes the proof.

We may now prove the main result of this section, that there are at least $p$ equivalence classes of $p$-coloured knots modulo surgery in $\operatorname{ker} \rho$, and that these are represented by connect-sums of left-hand $(p, 2)$-torus knots with a given colouring.

Proposition 19 For $r_{1} \neq r_{2} \bmod p$ the connect-sum of $r_{1}$ left-hand $(p, 2)$-torus knots with a given colouring is not equivalent modulo surgery in $\operatorname{ker} \rho$ to the connectsum of $r_{2}$ left-hand $(p, 2)$-torus knots with the same colouring.

Proof The proof is by comparing coloured untying invariants.

For $(K, \rho)$ a $p$-coloured left-hand $(p, 2)$-torus knot, $\widehat{C}_{2}$ is a $(1, p)$ lens space. Its fundamental group and therefore also its first homology is $\mathbb{Z} / p \mathbb{Z}$. Thus the $p$-colouring $\rho$ is an automorphism of $\mathbb{Z} / p \mathbb{Z}$ and therefore corresponds to a non-trivial cohomology class $a \in H^{1}\left(\widehat{C}_{2} ; \mathbb{Z} / p \mathbb{Z}\right)$. Since $\partial^{*} a$ is also non-trivial $\operatorname{cu}(K, \rho)$ is a non-trivial element of $H^{3}\left(\widehat{C}_{2} ; \mathbb{Z} / p \mathbb{Z}\right) \cong \mathbb{Z} / p \mathbb{Z}$.

The coloured untying invariant is additive, since taking the connect-sum of two knots corresponds to taking the direct sum of their Seifert matrices, and the characteristic link of the connect-sum may be taken to be the disjoint union of the characteristic link of the direct summands. An alternative proof of additivity is that for $K=K_{1} \# K_{2}$, the double covering $\widehat{C}_{2}$ decomposes as $\widehat{C}_{2}^{1} \oplus \widehat{C}_{2}^{2}$, and therefore $D(a)$ may be taken to be the disjoint union of two homology classes in $H_{2}\left(\widehat{C}_{2} ; \mathbb{Z} / p \mathbb{Z}\right)$. 


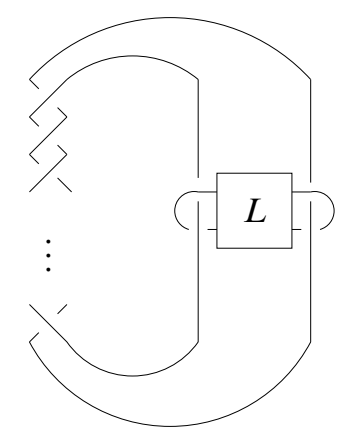

Figure 6: An untying link

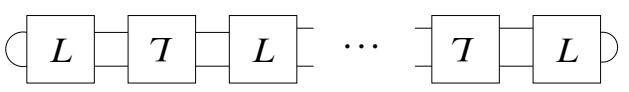

Figure 7: A surgery presentation for the irregular dihedral cover

\section{Applications}

\subsection{Surgery presentation for irregular dihedral coverings of $S^{3}$ branched over knots}

The original motivation for this paper was a series of discussions between Andrew Kricker and the author about finding surgery presentations for irregular branched dihedral coverings of knots.

Knowing how to reduce any $p$-coloured knot $(p=3,5)$ to a left-hand $(p, 2)$-torus knot (or a connect-sum of such) by surgery in the kernel of the $p$-colouring gives us a new algorithm for translating between covering presentations and surgery presentations of closed orientable 3-manifolds. First, by \pm 1 -framed surgeries in the kernel of its colouring, a $p$-coloured knot $K$ may be presented as a framed link in the complement of a connect-sum of $(p, 2)$-torus knots. Lifting the knot to the irregular branched dihedral covering space lifts also the link that is in its complement. Explicitly, in [9] we show that the surgery presentation of the irregular dihedral cover of a knot $K$ which is obtained from surgery by $L$ on the $(p, 2)$-torus knot as in Figure 6 (or a connect-sum of such) is given by Figure 7.

This method generalizes Yamada's algorithm [13], and does not require use of the 3 -move in the 3 -colour case.

Algebraic 83 Geometric Topology, Volume 6 (2006) 


\subsection{Surgery presentation of $D_{2 p}$-periodic maps on compact 3-manifolds}

Montesinos [8] showed that a closed orientable 3-manifold is a double branched covering of $S^{3}$ if and only if this manifold is obtained by rational surgery on a strongly invertible link $L$ in $S^{3}$. This allows us to 'visualize' the covering involution of such a manifold $M$, since it is conjugate to the involution of $M$ induced by the involution of $S^{3}$ preserving $L$.

This result has recently been generalized by Przytycki and Sokolov [10] and later by Sakuma [11] to all cyclic branched covering spaces. For a closed orientable 3manifold $M$ which admits an orientation-preserving periodic diffeomorphism $f$, Sakuma showed that $M$ is obtained by integral surgery on a link $L$ in $S^{3}$ which is invariant under a standard $\frac{2 \pi}{n}$ rotation $\varphi_{n}$ around a trivial knot, and $f$ is conjugate to the periodic diffeomorphism of $M$ induced by $\varphi_{n}$.

The key fact used in these papers which prevents the proofs there from directly carrying over to the dihedral case is that any knot $K$ can be transformed into an unknot by \pm 1 -surgeries on a trivial knot whose linking number with $K$ is 0 , but such surgeries may not be in the kernel of a $p$-colouring of the knot. As Makoto Sakuma pointed out to the author, by substituting our main theorem in this note for this fact, we may generalize the above result to the case in which the cyclic group is replaced by the dihedral group $D_{2 p}$ with $p \in\{3,5\}$ and in which $M$ is a regular dihedral $p$-fold covering space. It seems an interesting problem to generalize this result to a wider class of 3-manifolds.

\section{References}

[1] G Burde, H Zieschang, Knots, de Gruyter Studies in Mathematics 5, Walter de Gruyter \& Co., Berlin (2003) MR1959408

[2] S E Cappell, J L Shaneson, Invariants of 3-manifolds, Bull. Amer. Math. Soc. 81 (1975) 559-562 MR0367967

[3] S E Cappell, J L Shaneson, Linking numbers in branched covers, from: "Fourmanifold theory (Durham, NH, 1982)", Contemp. Math. 35, Amer. Math. Soc., Providence, RI (1984) 165-179 MR780578

[4] R H Fox, A quick trip through knot theory, from: "Topology of 3-manifolds and related topics (Proc. The Univ. of Georgia Institute, 1961)", (M K Fort, editor), Prentice-Hall, Englewood Cliffs, NJ (1962) 120-167 MR0140099

[5] S Garoufalidis, A Kricker, A surgery view of boundary links, Math. Ann. 327 (2003) 103-115 MR2005123 
[6] S Garoufalidis, A Kricker, A rational noncommutative invariant of boundary links, Geom. Topol. 8 (2004) 115-204 MR2033481

[7] A Kawauchi, A survey of knot theory, Birkhäuser Verlag, Basel (1996) MR1417494

[8] J M Montesinos, Surgery on links and double branched covers of $S^{3}$, from: "Knots, groups, and 3-manifolds (Papers dedicated to the memory of R H Fox)", Princeton Univ. Press, Princeton, N.J. (1975) 227-259. Ann. of Math. Studies, No. 84 MR0380802

[9] D Moskovich, A Kontsevich invariant for coloured knots, in preparation

[10] J H Przytycki, MV Sokolov, Surgeries on periodic links and homology of periodic 3-manifolds, Math. Proc. Cambridge Philos. Soc. 131 (2001) 295-307 MR1857121

[11] M Sakuma, Surgery description of orientation-preserving periodic maps on compact orientable 3-manfolds, Rend. Istit. Mat. Univ. Trieste 32 (2001) 375-396 (2002) MR1893406

[12] EH Spanier, Algebraic topology, McGraw-Hill Book Co., New York (1966) MR0210112

[13] T Yamada, Translation algorithms between the covering presentation and other presentations of 3-manifolds, Master's thesis, Tokyo Institute of Technology (2002)

Research Institute for Mathematical Sciences, Kyoto University

Kyoto 606-8502, Japan

dmoskovich@gmail.com

http://www. sumamathematica.com/

Received: 25 June 2005 